\title{
Advanced Safety Sensor for Gate Automation
}

\author{
Luca Bombini, Alberto Broggi, and Stefano Debattisti \\ VisLab - Dipartimento di Ingegneria dell'Informazione \\ Università degli Studi di Parma \\ \{broggi, bombini, deba\}@vislab.it \\ http://www.vislab.it
}

\begin{abstract}
This paper presents a vision-based method able to increase safety in access automation systems. These systems include automatic swing and sliding gates, bollards and barriers to prevent unwanted access. In current installations the anti-crushing protection is ensured by an electronic device installed on the control boards, which directly controls drive torque, and a couple of infrared photodetectors: when an obstacle is touched by the gate leafs or barriers, or cuts the infrared beam, the control board stops the gate movement. Conversely, the new method proposed in this paper avoids collisions with obstacles and increases the overall detection performance. The new device returns a stop signal when an obstacle is present in a predefined area. The algorithm has been integrated in a real access system to test its performance.
\end{abstract}

Keywords: safety sensor, gate automation control, obstacle detection, stereo vision.

\section{Introduction}

Access automation systems are used in many settings including residential or public areas, like park systems. They include automatic gates and barriers, swing and sliding gates, bollards and barriers to prevent unwanted access or to regulate traffic flow. The safety of these installations is a primary requirement considering their very large distribution in residential and commercial areas. Some of the possible hazards associated with this kind of automation are shown below:

* Crushing during closing.

* Shearing.

* Impacts.

* Person lifting.

* Entrapment.

In response to the latest European standards concerning safety in the access automation systems [6], industries developed a lot of products and accessories that comply with the latest European directives.

Despite that, these products have some key limitations due to the technology used: obstacle recognition is usually done with photodetectors, performing 
obstacle localization only along the straight line that connect transmitter to receiver; for this reason this kind of sensors can not cover the whole danger area. Another key limitation of photodetectors is that doors themselves must not be detected as obstacles, so photodetectors must be placed outside the danger area.

In order to increase the safety in access system, this paper shows a method able to meet the safety European specification and therefore avoid the hazards mentioned before. The goal of this work is to create a vision-based system able to detect obstacles in the danger area; in this way, the whole gate maneuver area can be monitored by the safety system.

The tests setup selected for the experimentation (see fig[1), include an automatic gate with two gate leafs. We have selected this scenario in order to deal with the most difficult case: two filled swing gate leafs that partially occluded the danger area in the opening/closing movement.

In order to obtain a robust system, several problems have to be analyzed and exploited:

- Different light conditions: the automatic gate access is placed outdoor and it has to work day and night; cameras can also be dazzled by the sun during the day.

- Vibrations: the mechanical movement of the doors can introduce vibrations in the gate pillars that would be transmitted to the cameras.

- Filled Doors: the danger area is partially occluded by the gate leafs.

- Camera positioning: the cameras are placed on the gate pillars (see Fig 1) in order to increase the field of view of the stereo systems. This positioning is unsual for stereo-based algorithms.

A very general setup has been studied to fit different kinds of automatic gates. A pair of CMOS cameras are mounted on the gate pillars overlooking the danger area. The cameras are connected to an embedded computer that processes the images and directly drives the access control board.

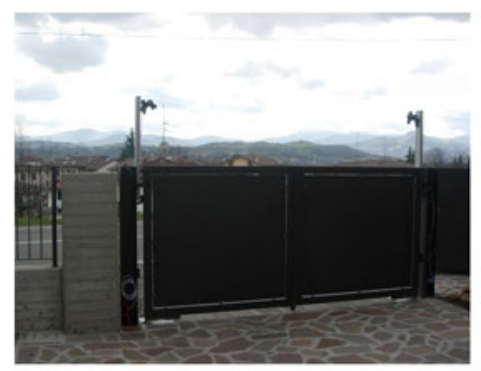

(a)

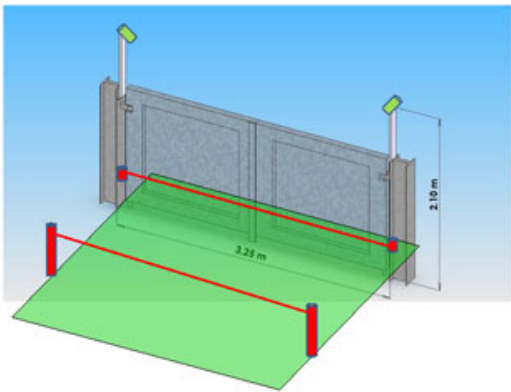

(a)

Fig. 1. (a) The camera installed on an automatic gate. (b) The cameras (green) are placed on the pillars and can replace the photodetector (red). The area monitored by the new vision-based system (green plane) is larger than the one covered by the photodetectors (red line). 


\section{The Algorithm}

The main goal of the application described in this paper is obstacle detection in a pre-defined area. Obstacle detection is a well known research field and several systems and solutions have been developed during the last years. The proposed approach differs from all the other existing systems because it takes in account the presence of a moving gate leafs in the analyzed scene. Therefore described algorithm will focus mainly on the innovative gate leafs detection system instead of the obstacle detection.

The gate leafs create two major problems:

- Obstacle Detection: gate leafs are not obstacles that need to stop the gate movement; we have to recognize them in order to avoid any possible obstacle false positive detection.

- Occlusion: we are working on a gate with filled swing-gate, so the gate leafs may occlude the cameras field of view.

In order to solve these problems, we have decided to create four virtual cameras, out of images coming from the two real cameras. The virtual cameras will be analyzed in the next subsection.

The whole algorithm is pictured in fig 2 and is performed in three main steps:

* Lens distortion and perspective removal from both images.

* Stereo Image Segmentation and creation of the images belonging to the four virtual cameras.

* Obstacle Detection.

Concerning the first step, during an offline preprocessing, a lookup table (LUT) that allows a fast pixel remapping is generated; this LUT associates each pixel in the distorted image to its homologous pixel on the undistorted image. Images of

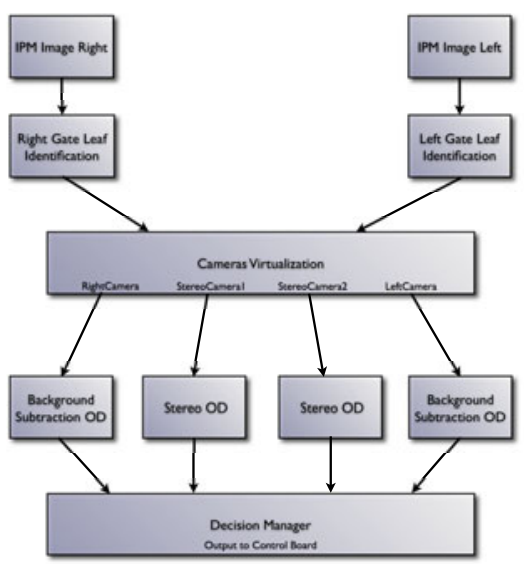

Fig. 2. Algorithm scheme 
a grid, painted on the danger area, are used to compute the LUT and a manual system to pinpoint all the crossing points on the source image is used. Thanks to the knowledge of the relative position of the cameras with respect to the grid itself and to the assumption that the danger area can be considered nearly flat, it is possible to compute a new image (the IPM image) removing both the perspective effect and camera distortion at once. A nonlinear interpolation function is used to remap the pixels of the source image that are not crosspoints [1.

Gate leafs recognition is performed next, as described in section 2.2. Recognizing the exact angular position of both gate leafs allows image segmentation in different zones with different characteristics. In particular the images have been segmented in stereo and mono zones, representing image areas visible from both cameras or only one camera.

Once images have been segmented, the algorithm performs camera virtualization: for each image area that will be analyzed by the obstacle detection algorithm, a virtual camera is created and the obstacle detection is executed directly on the images coming from this virtual sensor. In particular, as we will see in section 2.3. different approaches on obstacle detection have been used for different virtual cameras, due to the different information provided by each virtual sensor.

Therefore, obstacle detection based on background subtraction is applied to the images coming from the mono virtual cameras, while an obstacle detection algorithm that exploits the difference between IPM images is applied to the images coming from the stereo virtual cameras.

Each obstacle detection algorithm provides an obstacles map of the analyzed area as output, with some other information like obstacle position and size. These maps are then merged by a decision manager (DM) in order to provide a unique command to the gate control. In particular the DM analyzes and compares information provided by available maps according to the status of the gate as explained in table 1. The virtual camera names of table 1 are explained in section 2.1.

Table 1. Decision Manager

\begin{tabular}{|c|c|l|}
\hline GateStatus & Active Virtual Sensors & Note \\
\hline Opening & LC/RC, SC1 & $\begin{array}{l}\text { Objects placed behind the gate opening } \\
\text { line are not considered as obstacles }\end{array}$ \\
\hline Closing & SC1, SC2 & $\begin{array}{l}\text { Objects placed in front of the gate opening } \\
\text { line are not considered as obstacles }\end{array}$ \\
\hline Stopped & LC, RC, SC1, SC2 & $\begin{array}{l}\text { All the surveilled area is considered as dan- } \\
\text { gerous }\end{array}$ \\
\hline
\end{tabular}

\subsection{Virtual Sensors}

As seen in fig 2, the second step of the algorithm is image segmentation and virtual cameras creation.

In fig 3 a we can frame a typical gate-moving situation: the gate leafs are opening and the two cameras can not entirely see the safety zone. 


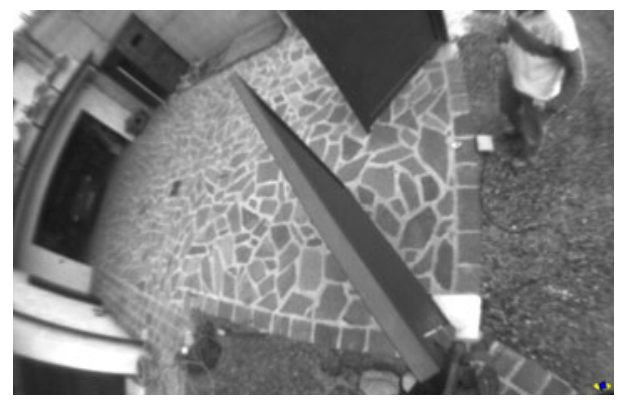

(a)

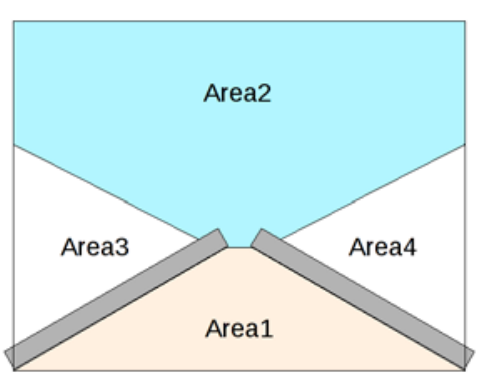

(b)

Fig. 3. Image from camera Left (a) and danger area segmentation (b)

The basic idea of our image segmentation is to split the original images in several sub-images with different characteristic, as we can see in fig 3 b.

The areas pictured in fig 3 , b require a different analysis: Area1 and Area2 are always visible from both cameras, while Area3 and Area 4 are visible from only one camera during the gate opening movement. Therefore, the following 4 virtual cameras are created:

- LeftCamera (LC): includes the Area3 image zone by segmenting the images coming from the left camera.

- Right Camera (RC): includes the Area4 image zone by segmenting the images coming from the right camera.

- Stereo Camera 1 (SC1): includes the Area1 image zone by segmenting the images coming from both cameras.

- Stereo Camera 2 (SC2): includes the Area2 image zone by segmenting the images coming from both cameras.

Each virtual vision system provides images to a specific obstacle detection algorithm.

\section{$2.2 \quad$ Gate Leafs detection}

At the base of the Gate Leafs Detection System (GLDS) there is the knowledge of the gate leafs' measures: starting from these values a mathematical model of each gate leafs is created, typically as a regular parallelepiped. The projection of the mathematical model on the IPM images coming from the calibrated cameras reduces the gate leafs detection problem to the detection of the gate leafs' angular position. Angular position may be detected using an artificial vision algorithm or using an encoder integrated on leafs electric motors. The encoderbased solution is safer than the other one, and is generally more precise; on the other hand using an encoder require a dedicated hardware component. For this reason, an artificial vision algorithm has been developed, but, because of the 
great differences between different types of gates, this algorithm is suitable only for systems described in Sec. 11. The main steps of GLDS are shown below:

- Background subtraction and binarization:moving objects are extracted from the images.

- Detection window reduction: the size of the images on which the detection will be performed is reduced, mantaining only the interesting areas.

- Comparation with gate leafs model: the objects found in the images are compared with the gate leafs mathematical model in order to estimate their angular position.

- Tuning of the detection: the angular position is refined using some assumptions.

The first step of the algorithm creates binarized images highlighting the objects moving in the scene; to do this, a standard background subtraction algorithm 2. has been used. The images coming from this step are next filtered with a Sobel filter and then binarized. In order to reduce the detection window we made some assumptions: we assume the gate trajectory as continuos, although not perfectly predictable, and the gate leafs velocity as quasi-constant. With these assumptions, and knowing the gate leaf position in the last frame, we are able to estimate the current gate leaf position; then we can consequently reduce the detection window to a sub-image, closer to the position we predicted. The detection window reduction brings several advantages: first, algorithm processing time improves, since the most complex part of the algorithm is represented by the comparison of the detection window with the mathematical gate leafs model; furthermore the reduction of the detection window size avoids many possible false positive detections, because the main component of the resulting image is represented by the gate leafs' edges. The output of this step of the algorithm is represented by an image, called detection window, containing the edges of the objects moving in the scene; to find out the position of the gate leafs we compare this image with the mathematical model of the gate. To do this, all the white pixels contained in the parallelepiped that represents a gate leaf are accumulated; this value represents a performance index of the correctness of the estimated angular position. This operation is repeated within a range of about 10 degrees with a step of 1 degrees, as we can see in fig 4
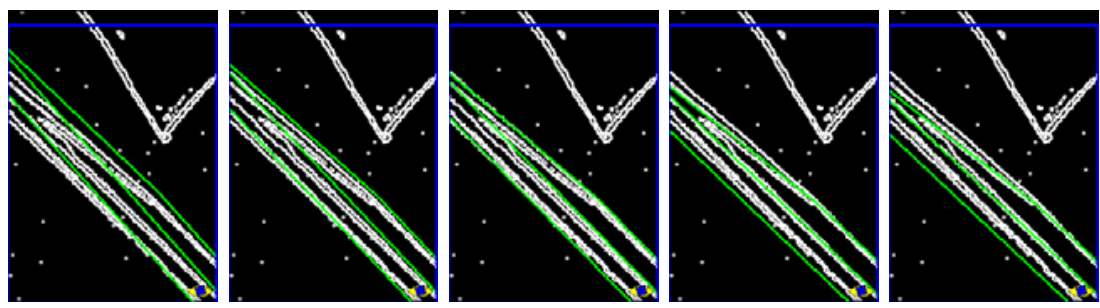

Fig. 4. GLDS: left gate leaf identification. A performance index is computed for each angular position; the estimated angular position is the one with the best index. 
It needs to be noted that we compare the left gate leaf model with the pixels coming only from the left image and the same for the right one; we perform these operations only if a correct position in the last frame was found. The last condition avoids wrong behaviors, like following wrong objects or an extra enlargement of the detection window. The verification of the correctness of the estimated angular position is based on the following assumptions:

- Quasi-constant gate leafs' velocity: the difference between the actual velocity and the velocities detected in the past frames must be lower than a given threshold.

- Movement consistency: both gate leafs must perform the same movement (opening, closing, stopped).

- Gate leaf priority: one of the gate leaf is always the first that opens and the last that closes, showing a relationship between the relative position of the two gate leafs.

In order to use these assumptions we compute the velocity of a gate leaf as the angle covered during the acquisition of an image frame, and the velocity error as the difference between the current velocity value and the average of the velocities computed over the last frames. If the estimated angular position verifies all three assumptions, their value is considered correct and the algorithm continue, otherwise an error signal will be sent to the gate control board.

\subsection{Obstacle Detection}

In our application two obstacle detection (OD) algorithms are used: background subtraction based OD and stereo based OD. The background subtraction based OD that we implemented is a simple algorithm that works on grayscale images and uses a basic motion detection[2] concept. The algorithm is based on the well-known assumption that a moving object is made of colors which differ from those in the background and can be summarized by the following formula:

$$
R_{i}= \begin{cases}255, & d_{i}>t \\ 0, & \text { otherwise }\end{cases}
$$

where $R_{i}$ is the $i$-th pixel of the resulting image and $d_{i}$ the difference of the same pixel in image $I$ and background image $B$, as expressed in this formula:

$$
d_{i}=\left|I_{i}-B_{i}\right|
$$

This algorithm takes as input the images coming from the virtual cameras called $L C$ and $R C$ and produces grayscale images containing the obstacles in the scene; the background image is updated only when the gate is closed and $S C 1$ and $S C 2$ do not retrieve any obstacles in the surveillance area.

The Stereo OD algorithm works on the images provided by $S C 1$ and $S C 2$ virtual cameras; starting from these IPM images, a difference image $D$ is generated comparing every pixel $i$ of the left image to its homologous pixel of the right one 
and computing their distance as in the background subtraction. The resulting image D is then filtered with a particular low pass filter that can be summarized by the following equation:

$$
\forall i \in D, \quad m=\frac{\sum_{\forall j \in A} D_{j}}{N_{A}} \quad T_{i}= \begin{cases}0 & m<y \\ 1 & m>y\end{cases}
$$

where $N_{A}$ represents the number of pixels in A.

This kind of low pass filtering is useful to find the main differences in these images and is faster than other similar methods[1]. The images coming from both OD algorithms are then labelled: each connected area is localized and labeled with a progressive number for further identification and filtering. The main difference between the two OD systems is that the stereo one is able to retrieve other additional information about the obstacles, like their position in the danger area, their size and their height; these information are useful for filtering and tracking making the stereo OD results more robust.

\section{Results}

The test activity was focused on the main differences between our new safety sensor and the standard safety systems for automatic gate control. To do this, several video sequences of a real gate system was taken; then these video sequences were processed offline by our algorithm. We created 5 tests, each one representing strengths or weaknesses of the traditional safety photodetector and for all of these tests we have then evaluated the behavior of our new system. The tests are structured as follow:

* Opening and closing without obstacles.

* Presence of obstacles detectable by photodetectors.

* Presence of obstacles not detectable by photodetectors because of their size.

* Presence of obstacles not detectable by photodetectors because of their position.

The aim of the first test was to evaluate the precision of the gate leaf detection algorithm and to check the robustness of the OD algorithms; the results were very satisfactory, the GLDS showed high precision and the OD assured good robustness, as shown in fig [5] where the output of the gate leaf detection system is highlighted by the green lines that compose the gate model. The second test was created to evaluate the effectiveness of our sensor when compared with to the traditional security sensor; our sensor outperformed the original setup because the Stereo OD performs a very precise obstacle detection. The third test covers a case in which the traditional photodetector fails: as shown in fig 6 the objects, in this case represented by some packages, were too small to be detected by the photodetector. Our safety sensor detects these objects in the correct position (as showed by the red marker of fig 6) regardless of their location or size. In fig 6. as in all figures shown in this section, the algorithm's output is represented 


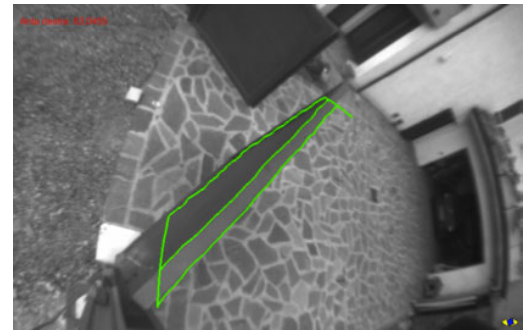

(a)

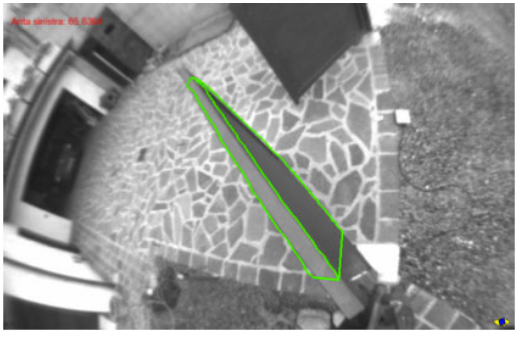

(b)

Fig. 5. Left (a) and Right (b) gate leaf identification

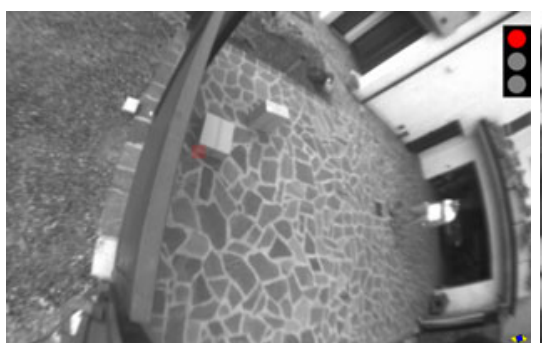

(a)

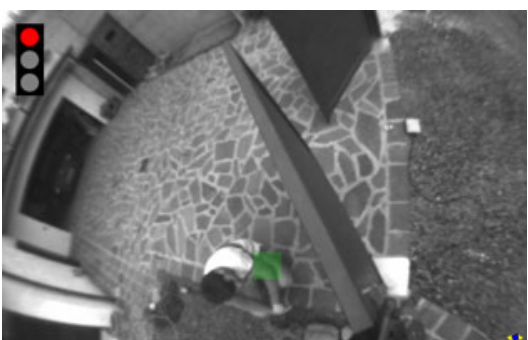

(b)

Fig. 6. Obstacles Detection: small size obstacles (a) and obstacles hidden behind a gate leaf (b)

by a traffic light that become green if the gate leafs can move or turns to red if they would hit an obstacle. The fourth test covers another case in which photodetectors do not work: in this case the obstacles location were such that they were not visible from the photodetectors. This test was also very useful to verify the strength of our background subtraction algorithm: obstacles were often visible only by $\mathrm{LC}$ or $\mathrm{RC}$, so they were visible only by the Background Subtraction based OD. Like in the other case, the results were extremely positive: the background subtraction algorithm provided enough robustness even with fast changing background and moving obstacles, as shown in fig 6 with a person hidden behind a gate leaf.

\section{Conclusion and Future Works}

In section 3 we saw that our advanced safety sensor showed satisfactory performance during the test stage: the sensor is able to detect obstacles in the whole danger area using different approaches for different zones of the danger area; furthermore the sensor showed an interesting robustness at the typical problems of outdoor vision system, as fast light changes, vibrations, wind and dazzle due 
to reflected sunlight. Despite that there are some situations that have to be thoroughly tested: i.e rain, snow and fog may request little changes in the obstacle detection algorithm and will be studied during the next months. Another interesting situation that we will take in to account in order to evaluate the system performances is the night scenario: neither the GLDS nor the $O D$ algorithm can work without natural sunlight, and the use FIR cameras must be excluded because of their cost. So, an artificial illumination system must be considered. The sensor described in this paper has patent pending.

\section{References}

1. Broggi, A., Medici, P., Porta, P.: StereoBox: a Robust and Efficient Solution for Automotive Short Range Obstacle Detection. EURASIP Journal on Embedded Systems (June 2007)

2. Benezeth, Y., et al.: Review and evaluation of commonly-implemented background subtraction algorithms. In: Pattern Recognition, ICPR (2008)

3. Bertozzi, M., Broggi, A., Fascioli, A.: Stereo inverse perspec- tive mapping: theory and applications. Image and Vision Computing 16(8), 585-590 (1998)

4. Collins, R.T., Lipton, A.J., Kanade, T.: A system for video surveillance and monitoring. Carnegie Mellon Univ., Pittsburgh (2000)

5. Bertozzi, M., Bombini, L., Broggi, A., Cerri, P., Grisleri, P., Zani, P.: GOLD: A Complete Framework for Developing Artificial Vision Applications for Intelligent Vehicles. IEEE Intelligent Systems (2008)

6. Informations on safe doors/gates, EN 12445 EN 12453 